\title{
AVALIAÇÃO DOS GASTOS COM MEDICAMENTOS UTILIZADOS NA ATENÇÃO BÁSICA DO RECIFE (2011 - 2015).
}

\author{
J. F. OLIVEIRA ${ }^{1}$, R.G. ARRUDA ${ }^{1}$, K. M. VILAR ${ }^{2}$, M. G. R PITTA ${ }^{2}$ \\ ${ }^{1}$ Centro de Ciências Sociais Aplicadas CCSA, Universidade Federal de Pernambuco \\ ${ }^{2}$ Núcleo de Pesquisa em Inovação Terapêutica Suely Galdino, UFPE \\ E-mail para contato: jfoliveir@hotmail.com
}

\begin{abstract}
RESUMO - Inserida no cenário da atenção básica, a Assistência Farmacêutica (AF) tem o papel de assegurar o acesso aos medicamentos à população, sendo parte fundamental para o restabelecimento da saúde e para a racionalidade de custos. O objetivo do estudo foi avaliar os gastos com medicamentos utilizados na atenção básica da cidade do Recife no período de 2011 a 2015. Foi realizado um estudo de natureza quantitativa, descritiva e retrospectiva referente aos gastos com os medicamentos utilizados nos serviços da atenção básica do Recife, através de estatística descritiva como análise tabular e gráfica, através do EXCEL®. A fonte dos dados foi a base de dados secundários da Assistência Farmacêutica do município (HORUS), do sistema de informação da atenção básica (SIAB) e do eSUS. Observou-se um incremento de $36 \%$ nos gastos com medicamentos que pode estar associado ao crescimento de outros fatores como o número de unidades de saúde, número de médicos, número de equipes de saúde da família e \% de cobertura da atenção básica. Nota-se que os medicamentos de uso contínuo foram responsáveis pela maior parcela dos gastos. O estudo tem como limitação o fato dos preços não terem sido deflacionados.
\end{abstract}

Palavras-chave: Assistência Farmacêutica. custos. atenção primária.

ABSTRACT - Inserted in the scenario of basic care, the Pharmaceutical Assistance (FA) has the role of ensuring access to medicines to the population, being a fundamental part for the restoration of health and cost rationality. The objective of the study was to evaluate the expenditures with drugs used in the basic care of the city of Recife in the period from 2011 to 2015. A quantitative, descriptive and retrospective study was carried out regarding the expenditures with the drugs used in the basic health services of Recife, through descriptive statistics such as tabular and graphical analysis, through EXCEL ${ }^{\circledR}$. The data source was the secondary database of the Municipal Pharmacy Assistance (HORUS), the basic care information system (SIAB) and e-SUS. There was a 36\% increase in drug costs, which may be associated with the growth of other factors such as the number of health units, number of doctors, number of family health teams and\% of coverage 
of basic care. It is noted that the drugs of continuous use were responsible for the greater part of the expenses. The study is limited by the fact that prices have not been deflated.

Key words: Pharmaceutical care. Costs. Primary care.

\section{INTRODUÇÃO}

A atenção básica (AB), como eixo ordenador da rede de atenção à saúde e coordenadora do cuidado, tem ampliado o acesso da população ao sistema público de saúde, buscando superar o modelo de saúde centrado na doença com a reorganização do processo de trabalho em saúde (MENDES et al., 2014; OLIVEIRA et al., 2010). Inserida nesse cenário, a Assistência Farmacêutica (AF) tem o papel de assegurar o acesso aos medicamentos à população, sendo parte fundamental para o restabelecimento da saúde e para a racionalidade de custos (ZULUAGA, 2013). Considerando a importância da Assistência Farmacêutica na resolutividade dos agravos à saúde, a escassez de recursos financeiros e a ampliação da rede de atenção básica na cidade do Recife, nos últimos anos, este estudo busca avaliar os gastos com medicamentos utilizados na atenção básica da cidade do Recife no período de 2011 a 2015

\section{MATERIAIS E MÉTODOS}

Estudo de natureza quantitativa, descritiva e retrospectiva referente aos gastos com os medicamentos utilizados nos serviços da atenção básica do Recife no período de 2011 a 2015, através de estatística descritiva como análise tabular e gráfica, através do EXCEL ${ }^{\circledR}$. A fonte dos dados foi a base de dados secundários da Assistência Farmacêutica do município (HORUS), do sistema de informação da atenção básica (SIAB) e do e-SUS.

\section{RESULTADOS E DISCUSSÃO}

Conforme mostra a figura 1, o gasto com medicamentos evoluiu no período, de forma não linear, e com considerável redução em 2012 e 2015, representando uma queda em torno de $18 \%$ em ambos os períodos. Como a maioria da população atendida no serviço público de saúde é de baixa renda, o fornecimento gratuito, é a única forma de acesso a medicamentos, por isso um a redução na aquisição dos medicamentos, pode favorecer o abandono do tratamento, com agravamento da condição de saúde e, consequentemente, aumento dos gastos com internações hospitalares (HELFER et al, 2012). 
Figura 1 - Gastos com medicamentos da atenção básica da cidade do Recife (20012015).

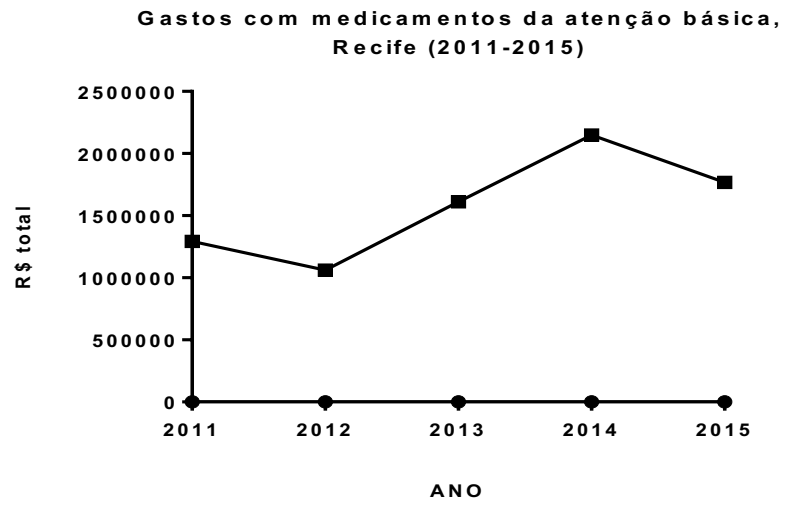

Observa-se então um crescimento no gasto com medicamentos com um incremento de 36\% (2011-2015), possivelmente associado a elevação dos indicadores descritos na tabela 1 .

Tabela 1 - Indicadores da atenção básica, Recife.

\begin{tabular}{lcc}
\hline Indicadores & $\mathbf{2 0 1 1}$ & $\mathbf{2 0 1 5}$ \\
\hline Unidades de saúde & 143 & 158 \\
$\mathrm{~N}^{\mathrm{o}}$ de profissionais médicos & 245 & 267 \\
$\mathrm{~N}^{\mathbf{0}}$ de equipes de saúde da família & 252 & 271 \\
$\begin{array}{c}\text { \% de cobertura da atenção básica } \\
\text { Fonte:Secretária de Saúde/Recife }\end{array}$ & 60 & 62
\end{tabular}

Ao analisar a evolução da assistência farmacêutica no contexto da atenção primária em saúde no município do Rio de Janeiro, Silva et al (2016) registraram um aumento dos gastos médios com medicamentos da atenção básica por habitante (20082014), ultrapassando o valor pactuado per capita a partir de 2013, bem como uma evolução de $128 \%$ do financiamento da assistência farmacêutica na atenção básica, índice superior ao crescimento de medicamentos hospitalares, cuja taxa foi de $29 \%$.

A figura 2 demonstra que os medicamentos do programa clínico, composto expressivamente por classes terapêuticas usadas em condições agudas, apresentam uma maior participação nos gastos totais, o que pode ser atribuído ao maior número de medicamentos cadastrados no programa. Os medicamentos usados em condições crônicas como doenças cardiovasculares, asma e saúde mental compõem expressivamente a maior participação relativa na composição dos gastos. Em sua análise dos gastos com medicamentos do componente básico da Assistência Farmacêutica no município de Jaboatão do Guararapes, Santos (2014) verificou que os subgrupos terapêuticos com maior impacto nos gastos foram os antibacterianos, anti-hipertensivos, anticonvulsivantes e hipolipemiantes. 
Figura 2 - Participação dos programas de saúde na composição dos gastos com medicamentos da atenção básica do Recife (2011-2015).

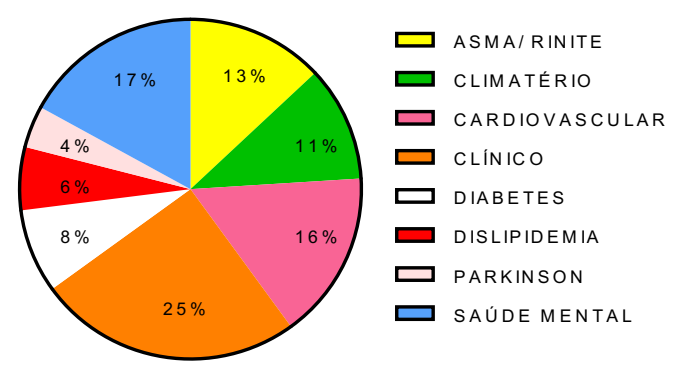

Apesar do orçamento público limitado, o custo dos medicamentos tem aumentado ao longo dos anos, repercutindo de forma significativa no campo da saúde pública (AMARAL; BLATT, 2011; TELES; COELHO, 2011). Alguns fatores têm contribuído para o aumento desses gastos como o aumento do número de idosos na população, ocasionado pela diminuição das taxas de fecundidade, natalidade e mortalidade infantil e pelo aumento da expectativa de vida, a transição das doenças infecciosas e parasitárias para as doenças crônicas não transmissíveis, a ampliação do acesso aos serviços de saúde e das listas de medicamentos fornecidos, além do uso irracional de medicamentos (CUNHA, 2014).

\section{CONCLUSÃO}

Os resultados revelam que houve um incremento nos gastos com medicamentos no período estudado, embora também haja momentos de declínio. Os medicamentos de uso contínuo são responsáveis pela maior parcela dos gastos. $\mathrm{O}$ estudo traz como limitação o não deflacionamento dos preços.

\section{REFERENCIAS}

AMARAL, S.M.S.; BLATT, C.R. Consórcio intermunicipal para a aquisição de medicamentos: impacto no desabastecimento e no custo. Rev. Saúde Pública. São Paulo, v. 45, n. 4, p. 799-801, Agosto de 2011.

HELFER, A.P et al. Capacidade aquisitiva e disponibilidade de medicamentos para doenças crônicas no setor público. Rev Panam Salud Publica, Washington, v. 31, n. 3, p. 225-232, Março de 2012.

SANTOS, J.N. Análise do gasto público com medicamentos do componente básico da assistência farmacêutica do município do Jaboatão dos Guararapes: tendência 2008 a 2013. Recife, 2014. Dissertação (Mestrado em Gestão e Economia da Saúde) Centro de Ciências Sociais Aplicadas, Universidade Federal de Pernambuco.

SILVA, R.M et al. Assistência farmacêutica no município do Rio de Janeiro, Brasil: evolução em aspectos selecionados de 2008 a 2014. Ciência \& Saúde Coletiva, Rio de Janeiro, v. 21, n. 5, p. 1421-1432, 2016 